\title{
Button Keyboard: A Very Small Keyboard with Universal Usability for Wearable Computing
}

\author{
Hyunjung Kim, Minjung Sohn, Seoktae Kim, Jinhee Pak, and Woohun Lee \\ Korea Advanced Institute of Science and Technology, Daejeon, Korea \\ \{rroseoscar, sohnminjung, seoktaekim, Jenny808, woohun. lee\} @kaist.ac.kr
}

\begin{abstract}
This paper presents the Button Keyboard, a very small wearable keyboard with universal usability. The Button Keyboard has high wearability and social acceptance due to its remarkably small size. As it is roughly button sized $(33 \mathrm{~mm} \times 33 \mathrm{~mm})$, it can be worn on the body without any discomfort. In addition, it adapts a mobile QWERTY layout to improve text entry for general users without special training. After an evaluation of the text input performance, it was found that the keyboard provides sufficient text input speed and learnability in spite of its small size. Furthermore, a touch-sensitive keypad divides input states by recognizing finger motions. Therefore, the system can provide visual or auditory feed-forwards to users. Thus, users can confirm the key before they execute. This previewable feature makes the keyboard a highly usable example of a wearable computer, in that it reduces the focus of attention and frequency of error. Moreover, it has the potential to assist the disabled by providing effective and appropriate ranges of feed-forwards.
\end{abstract}

Keywords: Wearable computing, Text entry, Keyboard.

\section{Introduction}

The absence of proper text input devices prevents the expansion of wearable computing. Current user interface devices for wearable computers are often considered to be obtrusive and appear unusual. Therefore, the use of wearable computing devices is limited to only researchers and a few early adopters. To ensure universal use, keyboards in the wearable computing environment should have high wearability and social acceptance. The operation of the keyboard should also be easy for all users to learn without special training. A sufficient input speed and a low error rate are important factors for text entry as well.

Many concepts and prototypes of wearable keyboards have been proposed and commercialized. However, wearable keyboards that consider universal usability have not been well researched. To make wearable computing devices usable by all, the size of the keyboard should be carefully considered [1]. It should be minimized to allow high social acceptance and wearability while providing high learnability and sufficient text input performance. 


\section{Reducing the Size of Wearable Keyboard}

Keyboard size is a crucial factor in deciding the wearability and social acceptance. Wearability and social acceptance are higher with a smaller keyboard. However, the extent that the size can be reduced is limited, as the performance of a keyboard depends on the physical size of the operating part of the human body. Keyboards with key pitches under certain size result in lower text input performance and higher fatigue level compared to common keyboards [2]. Therefore, it is important to estimate the optimum point between text input performance and wearability.

A One-key Keyboard was suggested as a new minimized keyboard in our previous research by the authors [1]. This keyboard has only one key, which has the same mechanism of a desktop keyboard and provides tactile feedback. This was a significant reduction in size compared to earlier keyboards. With the One-key Keyboard, key selection is conducted using a touch screen on the top of the keyboard that detects the position of a finger. A QWERTY layout was selected to improve performance and learnability. To determine the size of the keyboard, wearability and social acceptance were tested using six different-sized keyboard models following a QWERTY layout. The results of the study showed that wearability and social acceptance were evaluated positively when a keyboard is smaller than $90 \mathrm{~mm} \times 45 \mathrm{~mm}$ which is the size of a business card. The One-key Keyboard was $70 \mathrm{~mm} \times 35 \mathrm{~mm}$ in size with $7 \mathrm{~mm}$ key pitches. In a performance test, the participants achieved $18.9 \mathrm{WPM}$ (SD: 2.0) with a $6.7 \%$ (SD: $2.7 \%$ ) error rate. Considering the very small size of the One-key Keyboard, the text input performance was extraordinary, as no additional instrument, such as a stylus, was used.

\section{Concept of Button Keyboard}

Button Keyboard is an extension of the One-key Keyboard. Although the One-key Keyboard showed good performance and usability, it was limited in that it was a wrist-worn keyboard. Users should carry the keyboard everywhere at all times, which prove to be a burden. Therefore, the focus was on minimizing the keyboard size to roughly button-size $(33 \mathrm{~mm} \times 33 \mathrm{~mm})$, which would allow it to be attached to clothes (see Fig. 1, left). This was thought to make the wearable keyboard more usable for everyday use. A mobile QWERTY layout [3] was adapted to provide high learnability (see Fig. 1, right).

Similar to PreSense [4], the Button Keyboard senses finger motions on the keypad by using a touch sensor array. This makes it possible to divide input states into key selecting, touching and pressing states. Therefore, the system can provide visual or auditory feed-forwards to users before pressing the key. Furthermore, this provides independent character selection thus avoiding entry segmentation problem. Finger motions on the keypad without pressing buttons can also be recognized as commands. The user can input a space key, a back space key and the enter key with finger gestures (see Fig. 2, left). 

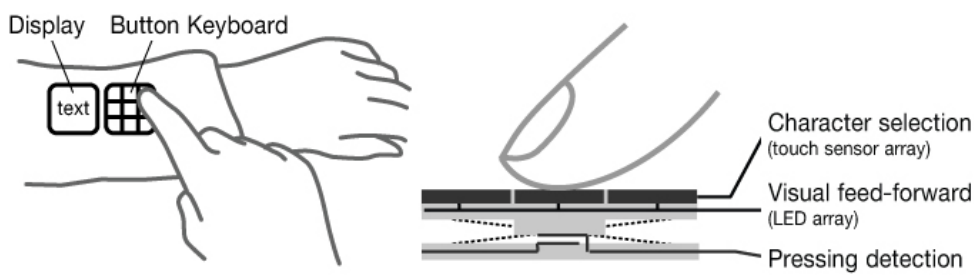

\begin{tabular}{|l|l|l|}
\hline EWQ & TUY & OP \\
\hline ADZ & RFV & ILJ \\
\hline SCX & HGB & NMK \\
\hline
\end{tabular}

Fig. 1. Concept of Button Keyboard (left: usage, center: structure, right: key layout)
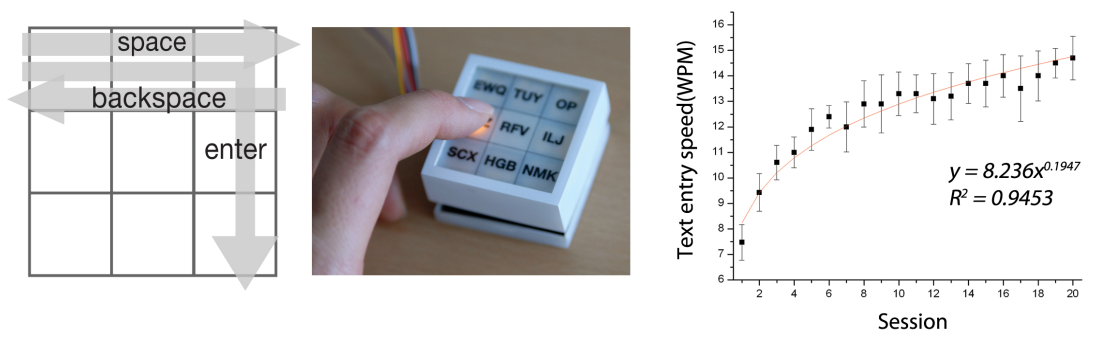

Fig. 2. Implementation and evaluation of Button Keyboard (left: finger gesture as commands, center: prototype, right: text entry speed of Button Keyboard)

\section{Evaluation of Button Keyboard}

In order to test the performance of the Button Keyboard, a text entry performance test was conducted. Ten college students (6 women, and 4 men; average age: 23.9) participated in the text-entry performance test of the Button Keyboard. The task was to enter short phrases of text taken from the Mackenzie et al. (2003) phrase set [5]. The subjects took part in twenty sessions lasting 10 minutes each.

On the 20th session, the entry speed was 14.7 WPM (SD: 1.70), and one of the participants achieved up to 17.0 WPM (see Fig. 4). The average error rate was $6.0 \%$ (SD: $0.66 \%$ ). In a comparison with results from other mobile phone keypads (15.5 WPM, 5.0\% error rate on the 20th session) [6] and considering that the participants are not native English speakers, the performance of the Button Keyboard is shown to be satisfactory. In addition, the participants used only one hand to input the text, which differs from how mobile phone keypads are used.

\section{Discussion and Conclusion}

The results of this study strongly suggest that the Button Keyboard provides sufficient text input performance in spite of its small size. It satisfies the requirements of high wearability, social acceptance, learnability and sufficient text input performance. Hence, the Button Keyboard is expected to be extremely useful in a wearable computing environment. In particular, the previewable feature of the Button Keyboard enables it to be used in various situations. For instance, it may be easier to 
use the Button Keyboard in a text input environment using a HMD (head - mounted display). The HMD will display keys to be pressed and the user will then be able to input text without increasing his or her focus of attention. In addition, the Button Keyboard is appropriate for disabled users. In particular, auditory feed-forwards for key inputting can facilitate text input for the people with limited sight. In situations in which users cannot see the keyboard, the auditory feed-forwards allow users to verify the corresponding character before typing it. Traditionally, the blind use a speechenabled typewriter. However, this device does not offer preview information; instead, it gives feedback only after typing. The utilization of finger gestures can also make key inputting, menu structure navigation and pointing much easier.

The next steps in this ongoing research include a field study. One of the limitations of this study is that all tests were performed in a lab environment. To ensure universal use of the Button Keyboard, it is necessary to test its usability with various types of users, including the different-abled users.

Acknowledgments. This work was supported in part by MIC \& IITA through the IT Leading R\&D Support Project and the BK21 program.

\section{References}

1. Kim, S., Sohn, M., Pak, J., Lee, W.: One-key Keyboard: A Very Small QWERTY keyboard Supporting Text Entry for Wearable Computing. In: Proceedings of OZCHI 2006, pp. 305 308 (2006)

2. Otsuka, I., et al.: Input Performance of One-Handed Keyboard. In: Proceedings of 7th Symposium on Human Interface, pp. 5-8 (1991)

3. Hwang, S., Lee, G.: Qwerty-like 3x4 keypad layouts for mobile phone. In: extended abstracts of the ACM conference on human factors in computing systems, pp. 1479-1482. ACM Press, New York (2005)

4. Rekimoto, J., Schewsig, C.: PreSense: Bi-directional Touch and Pressure Sensing Interactions with Tactile Feedback. In: CHI 2006 extended abstracts on Human factors in computing systems, pp. 1253-1258 (2005)

5. MacKenzie, I.S., Soukoreff, R.W.: Phrase sets for evaluating text entry techniques. In: extended abstracts of the ACM conference on human factors in computing systems, pp. 754-755. ACM Press, New York (2003)

6. Hwang, S., Lee, G.: Qwerty-like $3 \times 4$ keypad layouts for mobile phone. In: extended abstracts of the ACM conference on human factors in computing systems, pp. 1479-1482. ACM Press, New York (2005) 